\title{
Implementation of a Proactive Pilot Health Plan-Driven Opioid Tapering Program to Decrease Chronic Opioid Use for Conditions of the Back and Spine in a Medicaid Population
}

\author{
Julia Page, PharmD; Robin Traver, PharmD, CGP; Sital Patel, PharmD, MBA; \\ and Christopher Saliba, PharmD, MBA
}

\begin{abstract}
BACKGROUND: In 2016, the Oregon Health Authority and the Health

Evidence Review Commission implemented guidance for Oregon Medicaid members who were taking opioids for chronic pain related to conditions of the back and spine. This guidance required that an individualized taper plan be developed and initiated by January 1, 2017, and a discontinuation date for all chronic opioid therapy of January 1, 2018.

PROGRAM DESCRIPTION: This program evaluated the effect of a proactive and voluntary health plan-driven opioid tapering program on morphine equivalent daily dose (MEDD) before the implementation of governmental guidance. Two mailings were sent to the providers of the targeted members with a variety of resources to facilitate an opioid taper. Pharmacy claims were analyzed to measure member opioid use, in the form of MEDD, after the provider outreach to be compared with their MEDDs before the outreach.

OBSERVATIONS: A total of 113 members met the study inclusion criteria for the second provider outreach. Of the 19 members' providers who submitted responses via fax to the health plan in response to this outreach, 6 indicated they would initiate taper plans. Of the 6 members with taper plans, 5 had decreases in MEDD (3.6\%, 4.5\%, 42.9\%, 45.5\%, and 46.1\%) after the 3-month data collection period, while the sixth member had no change in MEDD. Of the 113 members, 16 members (14.2\%) had a decrease in MEDD; 23 members (20.4\%) had no change in MEDD; and 72 members $(63.7 \%)$ had an increase in MEDD.

IMPLICATIONS: This study demonstrated that when a physician agrees to enroll patients in a health-plan driven clinical program it may result in decreased opioid use as referenced by MEDD. However, the results also showed the progressive nature of opioid use in this population. While these initial taper results were promising, a larger sample size and longer followup duration are needed to validate long-term adherence to an opioid tapering program and confirm that these results are attributable to the program and not other factors.
\end{abstract}

J Manag Care Spec Pharm. 2018;24(3):191-96

Copyright $\odot 2018$, Academy of Managed Care Pharmacy. All rights reserved.

\section{What is already known about this subject}

Opioids are commonly prescribed for chronic pain yet have significant safety risks and are not recommended as first-line or routine therapy for this indication outside of the cancer or palliative care settings.

Regulatory bodies and health plans have developed initiatives to decrease opioid use and associated safety risks.

\section{What this study adds}

Health plan-driven clinical programs developed to proactively comply with governmental guidance regarding opioids may result in decreased opioid use.

An increase in opioid use may still occur despite health plan

intervention.

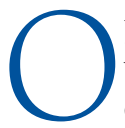
pioids are a commonly prescribed treatment for pain, with approximately 1 out of 5 patients with noncancer pain being treated with this class of medications. ${ }^{1}$ However, opioid therapy puts patients at risk for many serious harms, including opioid use disorder, opioid abuse, and opioid overdose. $^{2-5}$ The number of opioid deaths has grown greatly over the past 15 years, with a tripling of deaths from 1999 to $2014 .^{3}$ In 2015 alone, 52,404 deaths in the United States were attributed to drug overdoses, and $63.1 \%$ of those deaths involved an opioid. ${ }^{3}$ In Oregon, specifically, there is an average of 3 deaths per week from prescription opioid overdoses. ${ }^{6}$

Chronic pain, generally defined as pain lasting for greater than 3 months or past the time of expected tissue healing, affects over 100 million adults in the United States and accounts for a large percentage of opioid prescriptions. ${ }^{7,8}$ Over the past 2 decades, prescribers have been encouraged by many initiatives to improve pain treatment and identify pain as the "fifth vital sign."-12 Consequently, the number of opioid prescriptions written for chronic pain has increased substantially. ${ }^{8,11,12}$ This increase in prescriptions has been associated with the dramatic increase in opioid use, abuse, and death. . $^{8,9,12}$

In response to the opioid overdose epidemic, the Centers for Disease Control and Prevention developed guidelines in March 2016 to clarify recommendations for opioid prescribing in the setting of chronic pain outside of active cancer and palliative and end-of-life care. ${ }^{4}$ These guidelines outline treatment strategies for chronic pain, as well as benefits and harms of opioid therapy. ${ }^{4}$ Despite the large numbers of patients using opioid therapy, opioids are not recommended as first-line or routine therapy for chronic pain, since there is insufficient evidence of long-term (greater than 1 year) outcomes of opioid therapy for chronic pain. ${ }^{4,8}$ If opioid therapy is warranted, guidelines 
recommend balancing the benefits and harms carefully and combining opioid therapy with nonpharmacological and nonopioid pharmacologic therapies, as appropriate. ${ }^{4,5}$ Furthermore, it is recommended that benefits and harms should be evaluated continually-at least every 3 months-in order to taper opioids as necessary and prevent opioid use disorder. ${ }^{4}$

In response to recent opioid use and misuse, several health plan-driven initiatives have been published to help decrease unsafe opioid prescribing and usage..$^{12,13}$ In addition, pharmacy advocacy groups such as the Academy of Managed Care Pharmacy have held forums with the goal of improving pain management and preventing opioid use disorder. ${ }^{14}$ Regulatory bodies have also developed initiatives to address the opioid epidemic. ${ }^{11,15,16}$ One such example is the implementation by Colorado Medicaid of quantity limits on short-acting opioids in 2014. ${ }^{17}$

Specifically in the state of Oregon, the Oregon Health Authority and the Health Evidence Review Commission (HERC), which govern the Oregon Medicaid population, released new guidance on the chronic use of opioids relating to conditions of the back and spine in $2016 .{ }^{18}$ This guidance was shared through the Prioritized List, which is the Oregon Health Plan's method for allocating resources for health care. ${ }^{18,19}$ The Prioritized List ranks all health conditions based on clinical and cost-effectiveness and assigns a line number to each condition. ${ }^{20}$ Funding by the Oregon legislature then determines which lines or indications are covered..$^{20}$ Conditions that are on the Prioritized List may also have guideline notes, which provide additional information into what may or may not be covered on that line. ${ }^{18}$ In July 2016, Guideline Note 60, "Opioids for Conditions of the Back and Spine," outlined new transitional coverage for patients on long-term opioid therapy. ${ }^{18}$ This coverage required that patients have individualized taper plans by January 1, 2017, which included an end to opioid therapy of no later than January 1, 2018. ${ }^{18}$ Guideline Note 60 also referenced Guideline Note 56, "Non-Interventional Treatments for Conditions of the Back and Spine," to recommend noninterventional treatments for conditions of the back and spine, as well as noted that dependence and/or addiction related to opioids is considered a separate covered indication. ${ }^{18}$

To proactively comply with this new chronic opioid use coverage change from the HERC, an Oregon Coordinated Care Organization (CCO) implemented a voluntary opioid tapering program in October 2016 with the goal of safely decreasing opioid use in the affected population and transitioning to alternative therapies when appropriate.

\section{Program Description}

In October 2016, the health plan implemented an opioid tapering program for all CCO members for whom the guideline note applied. ${ }^{18}$ The total CCO member population at this time was approximately 45,000 members. Medical and pharmacy claims data were used to identify members for study inclusion. Members with medical claims diagnoses correlating to Guideline Note 60 (conditions of the back and spine) and paid pharmacy claims with a morphine equivalent daily dose (MEDD) were considered for inclusion in the study. ${ }^{18}$ Other inclusion requirements were greater than 90 days of a MEDD greater than 0 and a last opioid fill in August or September 2016. MEDD was calculated based on the opioid claim quantity multiplied by the appropriate MEDD conversion factor and then divided by the opioid claim days supply. Members with neoplasm medical claims were excluded.

\section{Program Phases}

The opioid tapering program consisted of the following 3 phases:

Phase 1: First Provider Outreach. The first provider mailing sent in early October 2016 included an introductory letter summarizing the HERC guidance and detailing the taper program..$^{18}$ The goal of this outreach was to familiarize any providers who had not been aware of the HERC guidance regarding the opioid tapering requirements. ${ }^{18}$ This mailing was sent to the primary CCO providers who were listed as such on members' registrations; however, these providers may not have prescribed the opioid prescriptions.

Phase 2: Second Provider Outreach. The second provider mailing sent in late October 2016 included a summary letter regarding the guidance, an example 10\% taper plan customized to the member's opioid regimen, a nonopioid analgesic therapy resource, a noninterventional therapy resource, and an "Opioid Tapering FAQ" patient handout. Providers were asked to return a response form to the health plan indicating their participation in the voluntary program or providing an explanation of why they declined to participate. The goal of this outreach was to obtain responses from providers regarding participation and to provide resources that would assist providers with realistically beginning safe and appropriate taper plans for their patients. In the majority of cases, this mailing was sent to the provider identified as a member's primary CCO provider on the CCO registration and who received the first mailing. For this second mailing, however, if the listed primary provider was not a single provider, such as in the case of a health system or clinic, it was sent instead to the provider who prescribed the most recent paid opioid claim for the member.

Phase 3: Data Collection and Analysis. Pharmacy claims from November 1, 2016, through January 25, 2017, were analyzed to measure member opioid use, in the form of MEDD, after the provider outreach, which would then be compared with members' MEDDs before the outreach. The change in the MEDD was evaluated to determine the effectiveness of the tapering program in decreasing member opioid use. Claims 


\section{FIGURE 1 Provider Outreach and Enrollment}

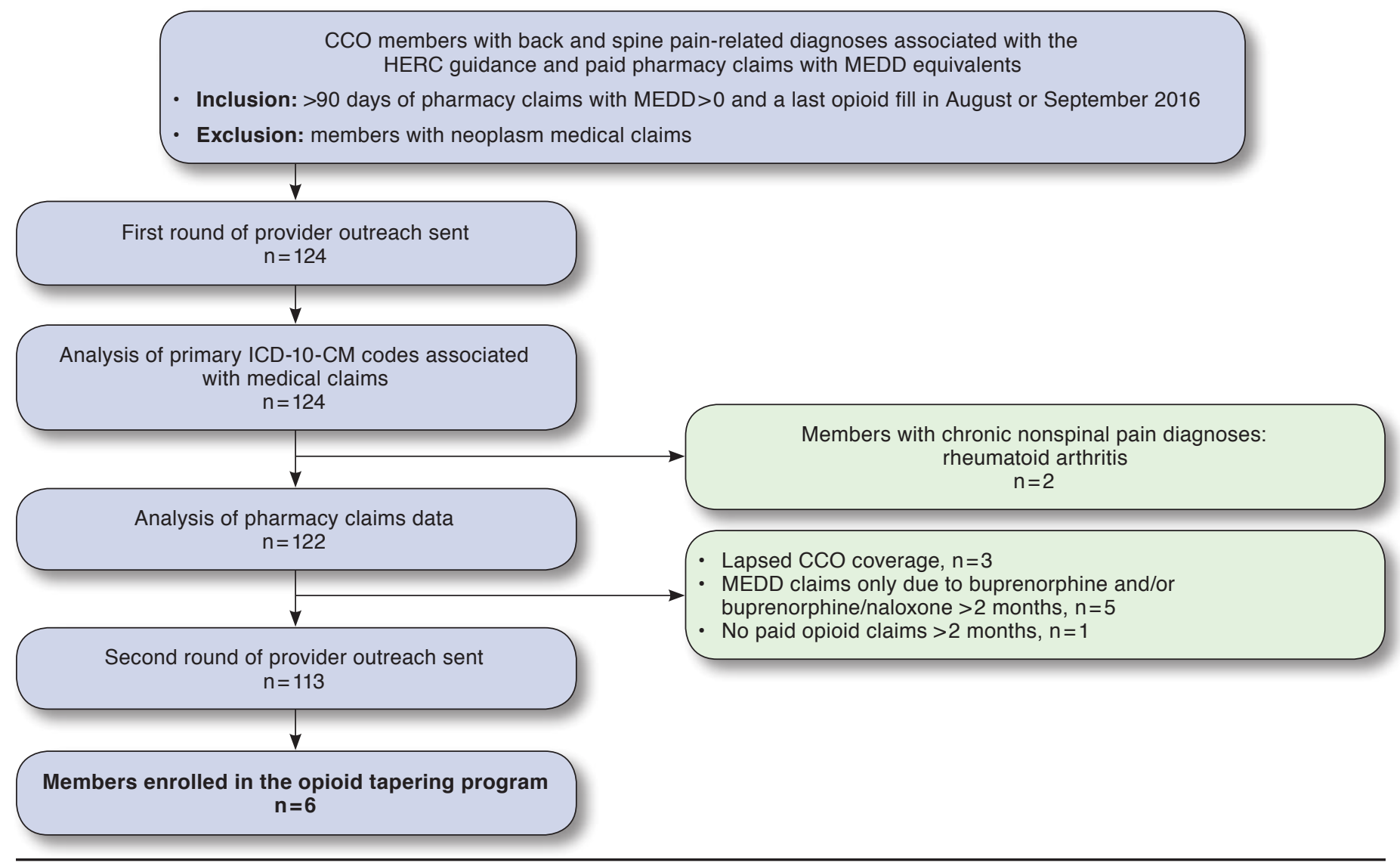

$C C O=$ Coordinated Care Organization; HERC=Health Evidence Review Commission; ICD-10-CM=International Classification of Diseases, Tenth Revision, Clinical Modification; $M E D D=$ morphine equivalent daily dose.

data were analyzed for all members who met inclusion criteria for the second provider mailing regardless of whether or not the providers indicated participation in the program.

\section{Observations}

A total of 124 members were identified for study inclusion in the first round of provider outreach based on a medical claims diagnosis related to Guideline Note 60 , as well as on pharmacy claims data with MEDDs greater than 0 for at least 90 days and a last fill in August or September 2016. ${ }^{18}$ Before the second provider outreach, however, further analysis of the 124 members was completed to exclude any members with medical claims associated with diagnoses of other causes of chronic nonmalignant nonspinal pain. The goal of this analysis was to eliminate any members who may have been taking opioids for diagnoses outside of chronic back and spine pain for whom the HERC guidance would not apply. After this analysis, 2 members were excluded from the study because of rheumatoid arthritis diagnoses.
Also, a pharmacy claims analysis was completed to eliminate any members for whom a tapering plan may not be appropriate. During this analysis, 9 additional members were excluded because of lapsed coverage, buprenorphine claims, or lack of opioid claims in more than 2 months. The flowchart in Figure 1 illustrates a more detailed view of included study members.

After the second round of outreach to the providers of the 113 members, 19 (17\%) responses were received by the health plan via fax. Of those 19 responses, providers for 6 (32\%) members documented that they would implement opioid taper plans with their patients; 5 (26\%) documented that their patients were being managed by a separate pain specialist; 5 (26\%) documented that taper plans were not appropriate for their patients due to medical reasons; and 3 (16\%) documented that taper plans were not appropriate for their patients due to miscellaneous reasons. Of the providers for the 113 members who received the second outreach, 94 (83\%) did not return a response via fax to the health plan. 


\section{FIGURE 2 Change in MEDD for All Members Involved in the Second Provider Outreach $(\mathrm{N}=113)$}

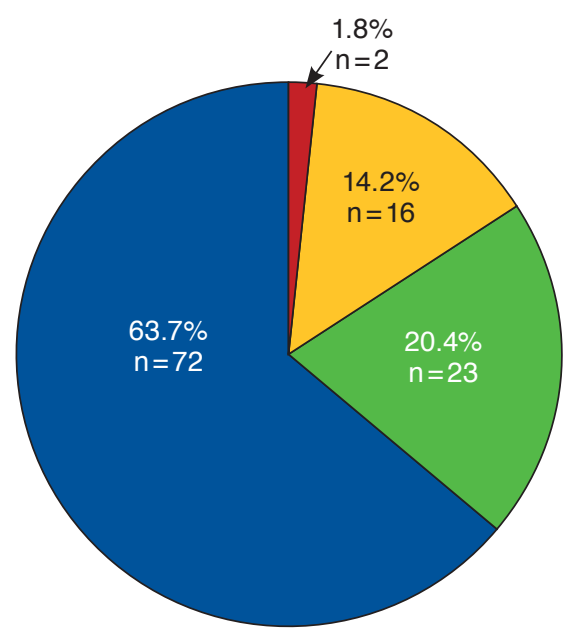

Decrease in MEDD No change in MEDD

Increase in MEDD

Unable to determine lapsed coverage

MEDD = morphine equivalent daily dose.

Before the tapering program, all 6 members whose providers documented that they would begin taper plans were on a combination of short- and long-acting opioids, and their preintervention MEDDs ranged from 110 to 1,020. Of those 6 members on taper plans, 1 had no change in MEDD after the 3-month data collection period. The other 5 members were found to have had a decrease in MEDD of 3.6\%, 4.5\%, 42.9\%, $45.5 \%$, and $46.1 \%$.

Of the 113 members, 16 members (14.2\%) had a decrease in MEDD; 23 members (20.4\%) had no change in MEDD; 72 members (63.7\%) had an increase in MEDD; and 2 members $(1.8 \%)$ were unable to be analyzed because of lapsed CCO coverage (Figure 2).

\section{Implications}

Opioids for chronic pain, while commonly prescribed and used, do not have long-term evidence supporting their efficacy. ${ }^{1}$ In addition, there are many safety risks associated with opioids, including opioid use disorder, opioid abuse, and opioid overdose..$^{2-5}$ Since opioid-related deaths and safety concerns have become topics of national concern, regulatory bodies have been increasingly outspoken about methods to address the opioid epidemic., ${ }^{3,11,15,16}$ The Oregon Health Authority's updated guidance for coverage of opioids in 2017-2018 represents another attempt at controlling the use of opioids in populations where safety and efficacy is not established. ${ }^{1,18}$ As more regulations continue to be created, health plans will need to determine the most effective ways of enforcing these initiatives for their members.

In this study, the interventions included 2 provider mailing outreaches that used multiple resources to assist providers in initiating realistic taper plans, when appropriate, for their patients. For the group of 113 members whose providers were mailed resources in the second mailing, the health plan received fax responses from 19 providers (17\%). Of those 19 responses, 6 (32\%) providers documented that they would implement taper plans for their patients. This low provider response rate may indicate that communicating with providers via mail and fax may not be a useful tool for promoting this type of clinical program. However, it may also indicate the hesitancy of prescribers to taper patients who are established on therapy or the difficulties that providers face in collaborating with patients to initiate an opioid taper schedule, including scheduling office visits for discussion and palliating any fears that the patient may have.

After the second outreach was made, claims data were collected from November 1, 2016, through January 25, 2017, to evaluate any change in member MEDDs. Of the 6 members whose providers indicated they would taper, 1 had no decrease in MEDD, while the other 5 had MEDD decreases of 3.6\%, $4.5 \%, 42.9 \%, 45.5 \%$, and $46.1 \%$ from their preintervention MEDDs, which ranged from 110 to 1,020 . In an additional analysis of these members' noninterventional therapies claims, it was found that only 1 member used an alternative therapy. The member with the 3.6\% MEDD decrease had claims for acupuncture during the data collection period. Of the 113 members included in the second outreach, 16 (14.2\%) had a decrease in MEDD. While this could potentially indicate that provider outreach may have had an effect on more members than the submitted responses indicated, it is not possible to determine if these results were because of the intervention or because of other factors, such as an increased emphasis on opioid tapering in the medical profession, political spotlight, and the media.

During that same data collection period (November 1, 2016-January 25, 2017), 23 members (20.4\%) had no change in MEDD, and 72 members (63.7\%) had an increase in MEDD. While some of the providers for these members may have discussed tapering or attempted to begin to taper these patients, these results indicate that provider outreach was not entirely successful in proactively complying with the Oregon Health Authority guidance. ${ }^{18}$ Reasons for this are unknown but may include difficulties in scheduling patients to initiate a taper schedule during this relatively short time frame; providers being overwhelmed by mail outreaches by health plans; and the nonurgent matter of the taper initiation due to the proactive time frame of the program compared with the deadlines in the regulatory guidance. 
Limitations of this study include the small sample size, lack of a comparator group, short duration, and low provider response rate. Also, the study population selected for this clinical program was based on pharmacy and medical claims data submitted between January 2016 and September 2016. Accuracy of the study population and results are dependent on claims being properly submitted. Gaps in percentage of days covered by opioid claims may also lead to the inaccurate appearance of MEDD and associated change in MEDD based on when the data were collected.

This study demonstrates that when a physician agrees to enroll patients in a health plan-driven opioid clinical program, it may result in decreased opioid use, as was seen with $14.2 \%$ of the members in this program. However, the results of this program also highlight the progressive nature of opioid use, since $63.7 \%$ of members ultimately had an increase in MEDD. While the initial taper results are promising, a larger sample size and longer duration of follow-up are needed to validate long-term adherence to an opioid tapering program and confirm that these results are attributable to the program and not other factors.

Areas for future program development include direct outreach to providers specializing in pain management for members who have their opioid medications prescribed at a pain clinic. Other types of outreach might also be considered, such as face-to-face provider discussions or forums, in order to enhance provider education and program adoption. In addition, as members continue to taper, more resources regarding opioid use disorder and opioid agonist therapies may be beneficial to providers. Finally, expanding the inclusion criteria to allow for a wider range of opioid claims data may result in a larger population, and continuation of the program through the governmental guidance implementation date would provide more robust results regarding the success of the program in complying with the opioid guidance.

\section{Authors}

JULIA PAGE, PharmD; ROBIN TRAVER, PharmD, CGP; SITAL PATEL, PharmD, MBA; and CHRISTOPHER SALIBA, PharmD, MBA, Pharmacy Services, Moda Health, Portland, Oregon.

AUTHOR CORRESPONDENCE: Julia Page, PharmD, Oregon State University College of Pharmacy, 2730 S.W. Moody Ave., CL5CP, Portland, OR 97201. Tel.: 503.494.4033;

E-mail:verhulst@ohsu.edu.

\section{DISCLOSURES}

This study was sponsored by Moda Health. Patel is employed by Moda Health; Page and Saliba were employed by Moda Health during this project; and Traver was employed by Moda Health during part of this project. Page is now employed by Oregon State University (during the writing of this manuscript) to support the College of Pharmacy's contract with the Oregon Health Authority to provide professional pharmacist support for the Oregon Medicaid program. All other authors have nothing to disclose.
Study concept and design were contributed by Page and Traver, who also collected the data. Data interpretation was performed by Page and Patel. The manuscript was written by Page and revised by Page, Patel, and Saliba.

\section{ACKNOWLEDGMENTS}

The authors thank Chelsea Keating, MPH, for her contributions to the analytics of this program, and Charles Hofmann, MD, for his contributions in the review of the program concept and design.

\section{REFERENCES}

1. Centers for Disease Control and Prevention. CDC guideline for prescribing opioids for chronic pain. March 16, 2016. Available at: https://www.cdc. gov/drugoverdose/prescribing/guideline.html. Accessed January 29, 2018.

2. Centers for Disease Control and Prevention. Guideline information for providers: safe prescribing saves lives. December 16, 2016. Available at: https://www.cdc.gov/drugoverdose/prescribing/providers.html. Accessed January 29, 2018.

3. Rudd RA, Seth P, David F, Scholl L. Increases in drug and opioid-involved overdose deaths-United States, 2010-2015. MMWR Morb Mortal Wkly Rep. 2016;65(5051):1445-52. Available at: https://www.cdc.gov/mmwr/volumes/65/wr/mm65505lel.htm. Accessed January 29, 2018.

4. Dowell D, Haegerich TM, Chou R. CDC guideline for prescribing opioids for chronic pain-United States, 2016. MMWR Recomm Rep. 2016;65(1):1-49. Available at: https://www.cdc.gov/mmwr/volumes/65/rr/rr650lel.htm. Accessed January 29, 2018.

5. American Pain Society, American Academy of Pain Medicine Opioids Guidelines Panel. Guideline for the use of chronic opioid therapy in chronic noncancer pain: evidence review. 2009. Available at: http://americanpainsociety.org/uploads/education/guidelines/chronic-opioid-therapy-cncp.pdf. Accessed January 29, 2018

6. Oregon Health Authority, Opioid Overdose and Misuse Public Health Division. Reducing opioid overdose and misuse. Available at: http://www. oregon.gov/oha/ph/PreventionWellness/SubstanceUse/Opioids/Pages/index. aspx. Accessed January 29, 2018

7. Institute of Medicine. Relieving Pain in America: A Blueprint for Transforming Prevention Care, Education, and Research. Washington, DC: The National Academies Press; 2011.

8. Chou R, Deyo R, Devine B, et al. The effectiveness and risks of long-term opioid treatment of chronic pain. Evidence Report/Technology Assessment No. 218. AHRQ Publication No. 14-E005-EF. September 2014. Agency for Healthcare Research and Quality. Rockville, MD. Available at: https://www. ncbi.nlm.nih.gov/books/NBK258809/. Accessed January 29, 2018.

9. Daubresse M, Chang HY, Yu Y, et al. Ambulatory diagnosis and treatment of non-malignant pain in the United States, 2000-2010. Med Care. 2013;51(10):870-78

10. Morone NE, Weiner DK. Pain as the fifth vital sign: exposing the vital need for pain education. Clin Ther. 2013;35(11):1728-32.

11. Surgeon General of the United States. Turn the TideRx: the Surgeon General's call to end the opioid crisis. Letter from the Surgeon General Available at: https://turnthetiderx.org/\#. Accessed January 29, 2018.

12. California Health Care Foundation. Changing course: the role of health plans in curbing the opioid epidemic. June 2016. Available at: https://www. chcf.org/wp-content/uploads/2017/12/PDF-ChangingHealthPlansOpioid. pdf. Accessed January 29, 2018.

13. Garcia MC, Dodek AB, Kowalski T, et al. Declines in opioid prescribing after a private insurer policy change-Massachusetts, 2011-2015. MMWR Morb Mortal Wkly Rep. 2016;65(41):1125-31. 
14. Proceedings of the AMCP Partnership Forum: breaking the link between pain management and opioid use disorder. J Manag Care Spec Pharm. 2015;21(12):1116-22. Available at: https://www.jmcp.org/doi/10.18553/ jmcp.2015.21.12.1116.

15. U.S. Department of Health \& Human Services. HHS takes strong steps to address opioid-drug related overdose, death and dependence. Press release. March 26, 2015. Available at: http://wayback.archive-it. org/3926/20170127185704/https://www.hhs.gov/about/news/2015/03/26/ hhs-takes-strong-steps-to-address-opioid-drug-related-overdose-death-anddependence.html. Accessed January 29, 2018.

16. Califf RM, Woodcock J, Ostroff S. A proactive response to prescription opioid abuse. N Engl J Med. 2016;374(15):1480-85.

17. Riggs CS, Billups SJ, Flores S, Patel RJ, Heilmann RMF, Milchak JL. Opioid use for pain management after implementation of a Medicaid shortacting opioid quantity limit. J Manag Care Spec Pharm. 2017;23(3):346-54. Available at: https://www.jmcp.org/doi/10.18553/jmcp.2017.23.3.346.
18. Oregon Health Authority, Oregon Health Evidence Review Commission. Prioritized List of Health Services. July 1, 2016. Available at: http://www. oregon.gov/oha/HPA/CSI-HERC/PrioritizedList/7-1-2016\%20Prioritized\%20 List\%20of\%20Health\%20Services.pdf. Accessed February 6, 2018.

19. DiPrete B, Coffman D. A brief history of health services prioritization in Oregon. Oregon Health Authority. March 2007. Available at: http://www. oregon.gov/oha/HPA/CSI-HERC/Documents/Brief-History-Health-ServicesPrioritization-Oregon.pdf. Accessed January 29, 2018.

20. Oregon Health Plan. The Prioritized List of Health Services. Available at: http://www.oregon.gov/OHA/HPA/CSI-HERC/Pages/Prioritized-List.aspx. Accessed February 6, 2018. 